\title{
PID Based on Attractive Ellipsoid Method for Dynamic Uncertain and External Disturbances Rejection in Mechanical Systems
}

\author{
Jesus Patricio Ordaz Oliver, ${ }^{1,2}$ Eduardo Steed Espinoza Quesada, ${ }^{2}$ \\ Abel Garcia Barrientos, ${ }^{1}$ and Julio Cesar Ramos Fernandez ${ }^{2}$ \\ ${ }^{1}$ Research Center on Technology of Information and Systems (CITIS), Autonomous University of Hidalgo State, \\ 42184 Mineral de la Reforma, Mexico \\ ${ }^{2}$ The Robotics and Advanced Electronics Research Laboratory, Polytechnic University of Pachuca, \\ 43830 Zempoala, HGO, Mexico
}

Correspondence should be addressed to Eduardo Steed Espinoza Quesada; steed@upp.edu.mx

Received 17 October 2014; Revised 21 January 2015; Accepted 21 January 2015

Academic Editor: Luis Rodolfo Garcia Carrillo

Copyright (C) 2015 Jesus Patricio Ordaz Oliver et al. This is an open access article distributed under the Creative Commons Attribution License, which permits unrestricted use, distribution, and reproduction in any medium, provided the original work is properly cited.

\begin{abstract}
This paper presents a stability analysis for LNDS (Lagrangian nonlinear dynamical systems) with dynamic uncertain using a PID controller with external disturbances rejection based on attractive ellipsoid methods, since the PID-CT (proportional integral derivative computed torque) compensator has been used for the nonlinear trajectory tracking of an LNDS, when there are external perturbations and system uncertainties. The global system convergence of the trivial solution has not been proved. In this sense, we propose an approach to find the gains of the nonlinear PID-CT controller to guarantee the boundedness of the trivial solution by means of the concept of the UUB (uniform-ultimately bounded) stability. In order to show the effectiveness of the methodology proposed, we applied it in a real 2-DoF robot system.
\end{abstract}

\section{Introduction}

The mathematical model for many physical (mechanical and electrical) systems is described by a set of nonlinear ordinary differential equations. Commonly, these mathematical models are described by a trajectory optimization procedure or by Lagrangian (Euler-Lagrange) dynamic equations [1, 2]. The main control problem for these systems involves some concepts of stability such as asymptotic, exponential, and uniform stability, among many others (see, e.g., [3-5]). Moreover, the high precision position and velocity control in Lagrangian systems are a fundamental and important control problem. In practice, the problem of tracking a reference trajectory where external disturbances, parameter uncertainties, or dynamical uncertainties are present has received relatively less attention in the literature. Traditionally, the methods to stabilize the Lagrangian systems are based on conventional $\mathrm{PD}$ (proportional derivative), $\mathrm{PD}+$ (PD with gravity compensation), PID (proportional integral derivative), and PIDCT compensators. It is well known that a PD controller can guarantee only asymptotic stability of Lagrangian systems in the regulation case. For the control theory viewpoint, it is well known that the steady-state error can be dissipated by introducing an integral compensator to the PD control or PID compensator. Nevertheless, in order to remove steadystate error caused by external disturbances, uncertainties, and noise, the integrator gain has to be increased. Nowadays, in industrial applications industrial linear PIDs are still used; however under certain circumstances, the stability of the closed-loop system with traditional PID is not guaranteed (from theoretical viewpoint). Thus, a high number of authors have been studying the global asymptotic stability around 
the origin of conventional compensators using the LNDS and the LaSalle's invariant principle theorem $[3,4,6,7]$. However, when the LNDS has unknown bounded external perturbations, the traditional analysis does not have a good efficiency. Besides, the transient performance and stability problems in the integrator theory analysis are also difficult to deal with for industrial linear PID controller. In order to ensure asymptotic stability of the PID controller, a traditional method is to modify the linear PID into a nonlinear one.

Nonetheless, it is well known that in many theoretical and practical applications the PID compensator, under satisfactory gain tuning, provides a workable trajectory enforcement. Moreover, from a certain point of view, the PID compensator, without full system description, achieves some robustness properties [6, 8-10]. Based on the above assumptions and after an exhaustive research in the area of classical closedloop controllers for LNDS we are inevitability addressed to the next question: under which conditions the classical PID is workable? Unfortunately, this is an open question because the classical PID compensator in presence of strong nonlinearities (like LNDS, tribology effects, external perturbations, or dynamic uncertainties) involves difficulties in dynamical analysis (see, e.g., [11, 12]). Even more, the qualitative operation of PID closed-loop solution of LNDS plays an important role in many engineering practical applications such as mechanical and power systems $[7,12,13]$. Although, for the classical linear PID, it is easy to prove asymptotic stability [11], it is not the case for the nonlinear system (this is the case for control analysis of LNDS or the Geometrical viewpoint (see [14, chapters 10-11] and [15])). So, in $[14,15]$ the authors established exponential stability only for the velocity error and feedforward compensator for LNDS, but this analysis does not tell us what happend with the external perturbations. In this work, we intend to define UUB-stability (for all system states) on the class of perturbed LNDS. Although PID control has been used in industrial robots for a long time, there is few explicit stability analysis on it. A wide variety of authors ensure asymptotic stability, but they do not take into account a robust analysis.

Nowadays, the development of high-performance robust control as well as its physical implementation is one of the main problems in the control theory, because in the experimental applications, we require a workable instrument to design compensators, which are able to operate successfully under consideration of external perturbations (see, e.g., [16-18]). This paper deals with the trajectory tracking control problem for LNDS, applied for a two-degree-offreedom (DoF) benchmark robotic manipulator. Here, we consider the PID-CT for LNDS with unknown but bounded external perturbations. This kind of perturbation belongs to the class of nonlinear functions so called quasi-Lipschitz functions. This class of functions permits to consider strong nonlinearities, like tribology effects and relay effects (such as the case when a robot task is to take an object from one point to another, which induces a switch on the parameters, e.g., mass and length to the center of mass). Even more, we suggest a robust analysis for a class of LNDS with bounded disturbances. Thus, in this paper we give an analysis of
UUB-stability for LNDS with unknown but bounded external perturbations from a class of quasi-Lipschitz functions.

The outline of this paper is as follows. In Section 2, we present a basic definitions and the problem formulation. Next section presents the main contribution of this work, based on the robust analysis of PID-CT compensator for Lagrangian systems. In Section 4, we obtain numerical results which define a two-link manipulator trajectory track. Finally, we present the conclusions.

\section{Basic Definitions and Problem Formulation}

In this section, we introduce the mathematical and LNDS mechanical tools needed for the remainder of this work.

\subsection{Lagrangian Dynamics}

2.1.1. Lagrangian Classical Model. The dynamic equation for mechanical or electrical systems can be obtained via the Newton's or Kirchhoff's laws approach, for $N$-DoF system or like in this case from the Lagrangian. These equations are obtained by the Lagrangian equation (for mechanical viewpoint). The general equation for the robot manipulator is given as

$$
\mathbf{D}(q) \ddot{q}+\mathbf{C}(q, \dot{q}) \dot{q}+G(q)=\tau,
$$

where the position coordinates $q \in \mathbb{R}^{n}$ with their associated velocities $\dot{q}$ and accelerations $\ddot{q}$ are controlled by the vector $\tau \in \mathbb{R}^{n}$ of driving forces. The Coriolis (centripetal) forces are $\mathbf{C}(q, \dot{q}) \dot{q} \in \mathbb{R}^{n}$, and the gravitational forces are denoted by $G(q) \in \mathbb{R}^{n}$. The dynamics (1) presents some interesting properties which will be useful in establishing the stability control analysis (see the Appendix).

2.1.2. Lagrangian Equations in First ODE Form. Observe that the general Lagrangian equation (1) can be represented in the first-order extended system as follows:

$$
\begin{gathered}
\dot{x}=f_{0}(x)+g(x) u, \\
x(0)=x_{0},
\end{gathered}
$$

where $x \in M \subseteq \mathbb{R}^{2 n}, u \in \mathbb{R}^{m}$ is the control input, and $x_{0} \subseteq$ $M$ are the initial conditions. From Lagrangian formulation under basic change of variables $x_{1}:=q \in \mathbb{R}^{n}, x_{2}:=\dot{q} \in \mathbb{R}^{n}$, $u:=\tau \in \mathbb{R}^{n}$, and $x=\left[x_{1}^{\top}, x_{2}^{\top}\right]^{\top}$, one has the mathematical model as

$$
\begin{gathered}
f_{0}(x)=\left[\begin{array}{c}
x_{2} \\
-\mathbf{D}^{-1}\left(x_{1}\right)\left\{\mathbf{C}(x) x_{2}+G\left(x_{1}\right)\right\}
\end{array}\right], \\
g(x)=\left[\begin{array}{c}
0_{n \times n} \\
\mathbf{D}^{-1}\left(x_{1}\right)
\end{array}\right] .
\end{gathered}
$$

2.1.3. The Uncertain External Perturbation. The external perturbations, affecting the system dynamics, are supposed to be bounded. By including this expression on the LNDS, we have

$$
\mathbf{D}\left(x_{1}\right) \dot{x}_{2}+\mathbf{C}(x) x_{2}+G\left(x_{1}\right)=\tau+\zeta(x, t),
$$


where the external perturbations have the following property:

$$
\zeta(x, t):=\zeta(q, \dot{q}, t), \quad\|\zeta(x, t)\|^{2} \leq c_{1}+c_{2}\|x\|^{2}
$$

for positive scalars $0<c_{1}, c_{2}<\infty$. Even more, it is admitted to have an unknown nonlinearity from a given class so-called quasi-Lipschitz functions [19]. Then, the nonlinearities $\zeta$ : $\mathbb{R}^{n} \rightarrow \mathbb{R}^{n}$ are from the $\mathscr{C}$ class of quasi-Lipschitz functions.

Definition 1 (the class $\mathscr{C}$ of quasi-Lipschitz functions [19]). A vector function $f_{1}: \mathbb{R}^{p} \rightarrow \mathbb{R}^{p}$ is said to be from the class $\mathscr{C}\left(\mathbf{A}, \delta_{1}, \delta_{2}\right)\left(\mathbf{A} \in \mathbb{R}^{p \times p} ; \delta_{1}, \delta_{2} \geq 0\right)$ of quasi-Lipschitzfunctions if for any $z \in \mathbb{R}^{p}$, it satisfies the inequality

$$
\begin{aligned}
\mathscr{C}\left(\mathbf{A}, \delta_{1}, \delta_{2}\right) & \\
:=\left\{f_{1}: \mathbb{R}^{p} \longrightarrow \mathbb{R}^{p} \mid\left\|f_{1}(z, t)-\mathbf{A} z\right\|^{2}\right. & \left.\leq \delta_{1}+\delta_{2}\|z\|^{2}\right\} \\
0 & <\delta_{1}, \delta_{2}<\infty .
\end{aligned}
$$

Notice that

(i) the growth rate of $f_{1}(z, t)$ as $\|z\| \rightarrow \infty$ is not faster than linear;

(ii) if $\delta_{1}=0$, the class $\mathscr{C}\left(\mathbf{A}, 0, \delta_{2}\right)$ is the class of Lipschitz functions.

This class of nonlinear functions may include discontinuous and hysteresis functions as well. Here, the perturbation $\zeta(x, t)$ under dynamics $\dot{x}_{2}$ has the following property:

$$
\begin{gathered}
f_{1}(x, t):=\mathbf{D}^{-1}\left(x_{1}\right) \zeta(x, t), \\
\mathbf{D}^{-1}(x) \leq \delta_{1} \mathbf{I}_{n \times n}
\end{gathered}
$$

which implies that $f_{1}(x, t) \leq \delta_{1} c_{1}+\delta_{1} c_{2}\|x\|^{2}$. The scalar, $\delta_{1}$, is given by the bounded property of the inertia matrix (see the Appendix). Finally, the Lagrangian equations in Cauchy form (2) in presence of external perturbations (4) have the following form:

$$
\dot{x}=f_{0}(x)+f_{1}(x, t)+g(x) u .
$$

In this work, we allow the external perturbations $f_{1}(\cdot)$ from a $\mathscr{C}$ class of quasi-Lipschitz functions. Now, our control objective is to stabilize (8) by using the PID-CT compensator.

2.1.4. PID-CT Configuration. In this work, the structure of the proposed control action $\tau$ is as follows:

$$
\begin{aligned}
u= & \mathbf{D}\left(x_{1}\right) \dot{x}_{2}^{d}+\mathbf{C}(x) x_{2}^{d}+G\left(x_{1}\right)-\mathbf{K}_{I} \xi\left(\tilde{x}_{1}\right) \\
& -\mathbf{K}_{P} \tilde{x}_{1}-\mathbf{K}_{D} \tilde{x}_{2}
\end{aligned}
$$

with the gain matrices $0<\mathbf{K}_{I}=\mathbf{K}_{I}^{\top}, \mathbf{K}_{D}=\mathbf{K}_{D}^{\top}, \mathbf{K}_{P}=\mathbf{K}_{P}^{\top} \epsilon$ $\mathbb{R}^{n \times n}$ and $\tilde{x}_{1}:=x_{1}-x_{1}^{d}, \tilde{x}_{2}:=x_{2}-x_{2}^{d}$ as dynamics error, where $x_{1}^{d}$ and $x_{2}^{d}$ are the deviation position and velocity, respectively, and $\xi(\cdot):=\int_{t_{0}}^{\top}(\cdot) d \tau$. In the classical robotics control strategies, the control action (9) is known as PID-CT compensator and it is well known that it has robustness properties.
2.2. Problem Formulation. In this section, we explain the problem formulation, which states that all system trajectories arrive to a neighborhood of a desired trajectory. This is by the following facts: When it is present noise in the position measurements (e.g., where the velocity and acceleration are estimated, by definition, from position measurement); possible change or switch in the system parameters, the time varying or like relay change on the parameters; external disturbances, the link contact with the external environment; dynamic uncertainties, Maxwell-slip friction model, Stribeck effect in sliding, frictional lag, varying break-away forces, stick-slip behavior, and among others tribology effects. For this reason, in this work, we approximate the error tracking path of the nonlinear system (8) to an AE (attractive ellipsoid).

Definition 2 (attractive ellipsoid). One says that the ellipsoid

$$
\mathscr{E}(0, \mathbf{P})=\left\{x \in \mathbb{R}^{n}: x^{\top} \mathbf{P} x \leq 1, \mathbf{P}=\mathbf{P}^{\top}>0\right\}
$$

(with the center in the origin and with the corresponding ellipsoidal matrix $\mathbf{P})$ is attractive for some dynamic system if for any of its trajectories $\{x\}_{t \geq 0}$ the following property holds:

$$
\limsup _{t \rightarrow \infty} x^{\top}(t) \mathbf{P} x(t) \leq 1
$$

Remark that all trajectories $\{x\}_{t \geq 0}$ of a dynamic system remain bounded, if for this system there exists an attractive ellipsoid $\mathscr{E}(0, \mathbf{P})$. The existence of an $\mathrm{AE}$ is the generalization of the UUB-property (uniform-ultimately boundedness) discussed in [5]. Our main goal is to design a robust feedback based on PID compensator; moreover, the compensator rejects disturbances without loss of tracking trajectories paths.

\section{On the UUB-Stability of the PID-CT for LNDS}

The robustness of the PID compensators on dynamical systems is a classical topic in control theory. Here, we present a stability sketch from LNDS in closed-loop with PID-CT. It is well known that the kinetic energy plays an important role for stability analysis for LNDS. In this paper, we use the energy function based on kinetic energy as follows:

$$
V(y)=\langle y, \mathbf{P} y\rangle, \quad y:=\left[\xi\left(\tilde{x}_{1}\right)^{\top}, \tilde{x}_{1}^{\top}, \tilde{x}_{2}^{\top}\right]^{\top},
$$

where the matrix $\mathbf{P}=\mathbf{P}^{\top} \in \mathbb{R}^{3 n \times 3 n}$ is a positive definite matrix and its structure is defined by $n \times n$ matrix subblocks as follows:

$$
\mathbf{P}:=\left[\begin{array}{ccc}
\mathbf{P}_{11} & \mathbf{P}_{12} & 0_{n \times n} \\
\mathbf{P}_{12}^{\top} & \mathbf{P}_{22} & 0_{n \times n} \\
0_{n \times n} & 0_{n \times n} & \mathbf{D}\left(x_{1}\right)
\end{array}\right], \quad \text { where } \mathbf{P}_{11}, \mathbf{P}_{12}, \mathbf{P}_{22} \in \mathbb{R}^{n \times n} .
$$

The following theorem presents the analysis for global stability of the PID-CT of system (8) under the specific energetic function (12). 
Theorem 3 (on the energy function of the PID-CT). If the tuning of the control gain matrices $\mathbf{K}_{P}, \mathbf{K}_{D}$, and $\mathbf{K}_{I}$ of the control action (9), under given matrix $\mathbf{P}$ and positive scalars $\alpha, \varepsilon$, is such that the following LMI constraint holds:

$$
0 \leq\left[\begin{array}{ccc}
\alpha \mathbf{P}_{11}-\varepsilon c_{1} \mathbf{I}_{n \times n} & \alpha \mathbf{P}_{12}+\mathbf{P}_{11}^{\top} & \mathbf{P}_{12}-\mathbf{K}_{I}^{\top} \\
\alpha \mathbf{P}_{12}^{\top}+\mathbf{P}_{11} & \alpha \mathbf{P}_{22}+\mathbf{P}_{12}+\mathbf{P}_{12}^{\top} & \mathbf{P}_{22}^{\top}-\mathbf{K}_{P}^{\top} \\
\mathbf{P}_{12}^{\top}-\mathbf{K}_{I} & \mathbf{P}_{22}-\mathbf{K}_{P} & \alpha \mathbf{A}-\mathbf{K}_{D}
\end{array}\right]
$$

then the energy function (12) has the following property:

$$
\begin{gathered}
\lim _{t \rightarrow \infty}\langle y, \overline{\mathbf{P}} y\rangle \leq 1, \\
\overline{\mathbf{P}}:=\frac{\alpha}{\beta}\left[\begin{array}{ccc}
\mathbf{P}_{11} & \mathbf{P}_{12} & 0_{n \times n} \\
\mathbf{P}_{12}^{\top} & \mathbf{P}_{22} & 0_{n \times n} \\
0_{n \times n} & 0_{n \times n} & \mathbf{A}
\end{array}\right], \quad \beta:=\varepsilon \mathcal{c}_{0} .
\end{gathered}
$$

The proof of all statements is given in the Appendix.

Remark 4. From Schur's complement it is easy to see that a necessary condition for the linear matrix inequality solution (14) provides positive definite matrix $\mathbf{P}$ for some $\mathbf{P}_{12}+\mathbf{P}_{12}<$ $-\alpha \mathbf{P}_{22}, \mathbf{P}_{11}<\varepsilon c_{1} / \alpha$, and $\alpha \mathbf{A}<\mathbf{K}_{D}$.

Notice that the extended trajectories $y(t)$ converge to an invariant ellipsoid $\mathscr{E}(0, \overline{\mathbf{P}})$ centered on the origin with ellipsoidal matrix $\overline{\mathbf{P}}$. In other words, $y(t) \rightarrow \mathscr{E}(0, \overline{\mathbf{P}})$ as $t \rightarrow \infty$. This ellipsoid is approached with the exponential rate $\exp (-\alpha t)$ fulfilling with

$$
\langle y(t), \overline{\mathbf{P}} y(t)\rangle-1 \leq\left(V(0)-\frac{\varepsilon}{\alpha} c_{0}\right) \exp (-\alpha t) .
$$

Moreover, notice that the selection of matrix $\mathbf{P}$ implies a family of possible ellipsoidal matrix configurations and may be selected in order to minimize the energy function (12). In further works, we will study this case from an optimization point of view [19].

Remark 5. It is well-known that the concept of an energetic function was rigorously formalized by means of the Lyapunov stability theory as well as the notion of a positive invariant set. Here, we just notice that the set of solutions $\left(\mathbf{K}_{P}, \mathbf{K}_{D}, \mathbf{K}_{I}\right.$, and $\mathbf{W}$ ) is given by Theorem 3 , such that (15) holds, and the storage function (12) is not obligatory monotonically nonincreasing. That means that $V(z)$ is not a Lyapunov function for the considered system at least for this timeinterval.

Below, we suggest the construction of a LyapunovLike function whose derivative on the trajectories of the considered controlled system is strictly negative outside of an ellipsoid. Which implies that any trajectory that begins outside the ellipsoid (15), this returns asymptotically within the ellipsoid. In other words, UUB-stability is guaranteed if the ellipsoid is an attractive region. The above is set to the next result.

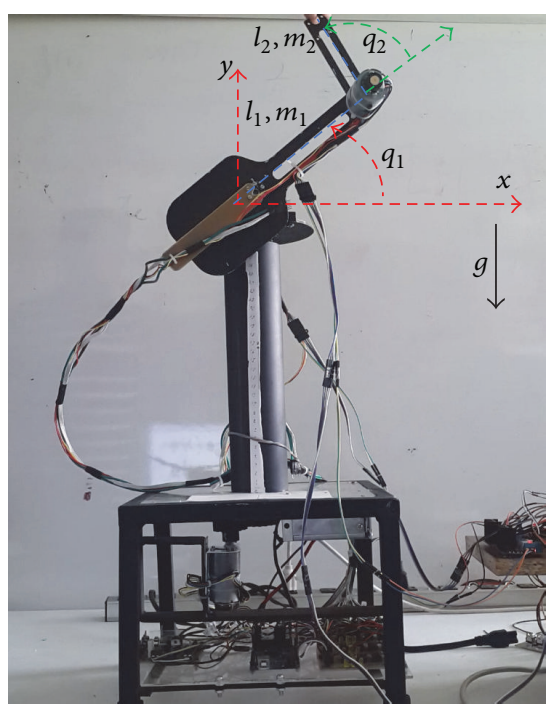

FIGURE 1: 2-DOF manipulator system.

Theorem 6 (on the attractively of the ellipsoid). Under the assumption of Theorem 3, the function

$$
\begin{gathered}
G(V(y)):=\left(\left[\sqrt{V(y)}-\sqrt{\frac{\beta}{\alpha}}\right]_{+}\right)^{2}, \\
{[\bar{y}]_{+}:= \begin{cases}y, & \text { if } y \geq 0, \\
0, & \text { if } y<0\end{cases} }
\end{gathered}
$$

is a Lyapunov function, and then the following property

$$
\frac{d}{d t} G(V(y)) \leq 0
$$

is globally satisfied, which exactly means that the set $\left\{y \in \mathbb{R}^{3 n}\right.$ : $G(V(y))=0\}$ is a positive invariant region.

Notice that the results of Theorem 6 involve the so-called UUB-property on the solution of the system trajectories (see [5]), satisfying the problem statement (15), so we may conclude that the PID-CT compensator, for a nonlinear system (8), is robust under external perturbations.

\section{Experimental Validation}

In order to show the contribution of the results obtained in this paper, we obtain the PID-CT gain matrices under two approaches. The first one is given by classical Ziegler-Nichols method (popular technique for tuning controllers that use PID actions [11, 12]), while the second one is given by LMI solution (14) introduced in this paper (see Theorem 3). The system to be controlled is a benchmark classical system (the second-link vertical planar robot shown in Figure 1, whose dynamics are given in the literature; see [7]). The model of the motion dynamics is a set of 2 rigid bodies connected and described by a set of generalized coordinates $q \in \mathbb{R}^{2}$. The derivation of the motion equations is given by (1) and by 

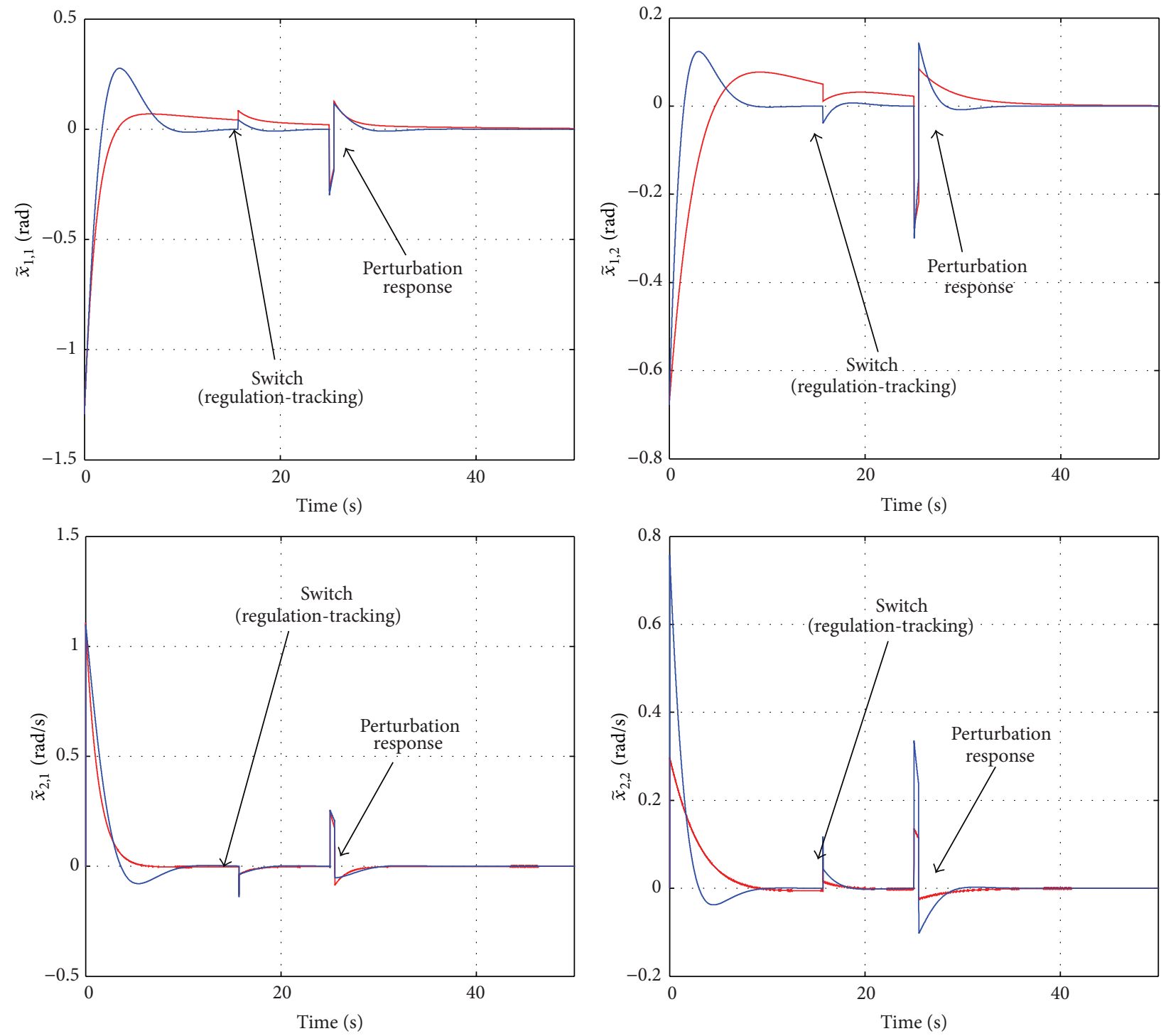

FIgURE 2: Error signals $\tilde{x}_{i, j}$ for $i, j=1,2$ of the trajectory tracking. The subindexes $i$ and $j$ are the position and velocity.

applying the methods of the Lagrange theory, involving explicit expressions of kinetic energy and potential energy, where we obtained the standard general equation (1). For this robot study case the position, velocity, acceleration, and control input $\left(q, \dot{q}, \ddot{q}\right.$, and $\tau$, resp.) belong in $\mathbb{R}^{2}$. Under the change of variable $\left(q_{1}, q_{2}, \dot{q}_{1}, \dot{q}_{2}\right):=\left(x_{1}^{\top}, x_{2}^{\top}\right)=x^{\top}, x_{1}^{\top}=\left(x_{1,1}\right.$, $\left.x_{1,2}\right)$, and $x_{2}^{\top}=\left(x_{2,1}, x_{2,2}\right)$, the nonlinear system described in the first-order Cauchy form (2) is given as follows:

$$
\begin{gathered}
\mathbf{D}\left(x_{1}\right)=\left(\begin{array}{cc}
\theta_{1}+\theta_{2}+\theta_{3} \cos \left(x_{1,2}\right) & \theta_{2}+\theta_{3} \cos \left(x_{1,2}\right) \\
\theta_{2}+\theta_{3} \cos \left(x_{1,2}\right) & \theta_{2}
\end{array}\right), \\
\mathbf{C}(x)=-\theta_{3} \sin \left(x_{1,2}\right)\left(\begin{array}{cc}
x_{2,2} & x_{2,1}+x_{2,2} \\
x_{2,1} & 0
\end{array}\right), \\
G\left(x_{1}\right)=\left(\begin{array}{c}
\theta_{4} g \cos \left(x_{1,1}\right)+\theta_{5} g \cos \left(x_{1,1}+x_{1,2}\right) \\
\theta_{5} g \cos \left(x_{1,1}+x_{1,2}\right)
\end{array}\right),
\end{gathered}
$$

$$
u=\left(\begin{array}{l}
u_{1} \\
u_{2}
\end{array}\right)
$$

Now the parameters of this manipulator (given in the international system of units) are $\theta_{1}=m_{1} l_{c 1}^{2}+m_{2} l_{1}^{2}+\square_{1}, \theta_{2}=m_{2} l_{c 2}^{2}+\mathbb{1}_{2}$, $\theta_{3}=m_{2} l_{1} l_{c 2}, \theta_{4}=m_{1} l_{c 1}+m_{2} l_{1}$, and $\theta_{5}=m_{2} l_{c 2}$, with the total mass of link- $1 m_{1}=0.5 \mathrm{~kg}$, the total mass of link- $2 m_{2}=$ $0.25 \mathrm{~kg}$, the moment of inertia of link- $1 \rrbracket_{1}=0.004 \mathrm{Kg} \cdot \mathrm{m}^{2}$, the moment of inertia of link $-2 \mathbb{~}_{2}=0.005 \mathrm{Kg} \cdot \mathrm{m}^{2}$, the distance to center of mass of link-1 $l_{c 1}=0.275 \mathrm{~m}$, the distance to center of mass of link- $2 l_{c 2}=0.1 \mathrm{~m}$, the length of link- $1 l_{1}=0.4 \mathrm{~m}$, the length of link- $2 l_{2}=0.2 \mathrm{~m}$, and the acceleration of gravity constant $g=9.81 \mathrm{~m} / \mathrm{seg}^{2}$. The distance to center of mass is defined by $l_{c 1}$ and $l_{c 2}$, respectively. Here, we consider that the dynamics are given by

$$
\mathbf{D}\left(x_{1}\right)+\mathbf{C}(x) x_{2}+G\left(x_{1}\right)+\mathbf{F}_{r}(x)=\tau+\xi(t),
$$



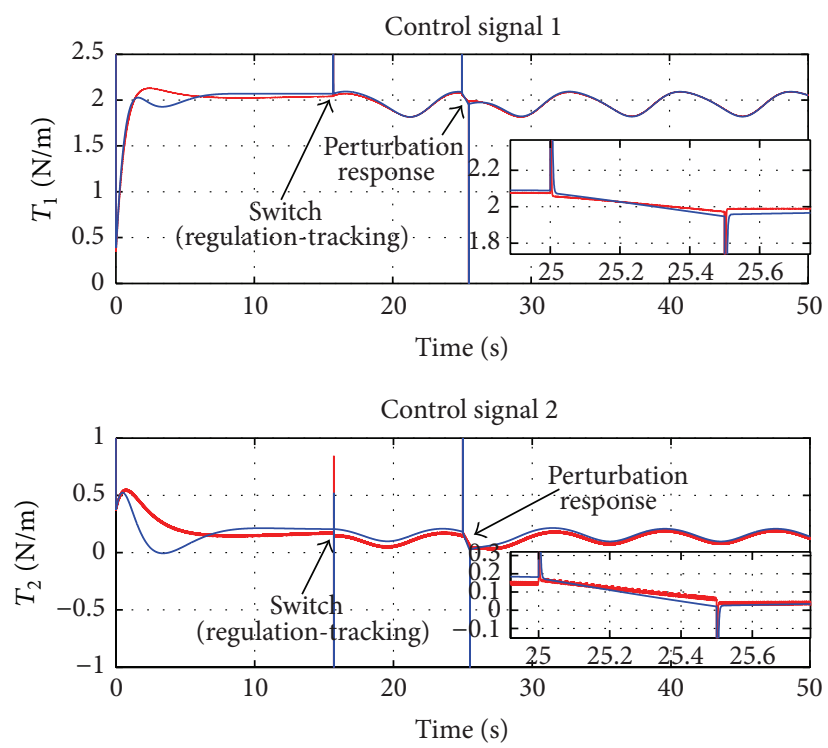

FIGURE 3: Control signals, for first and second link (two algorithms).
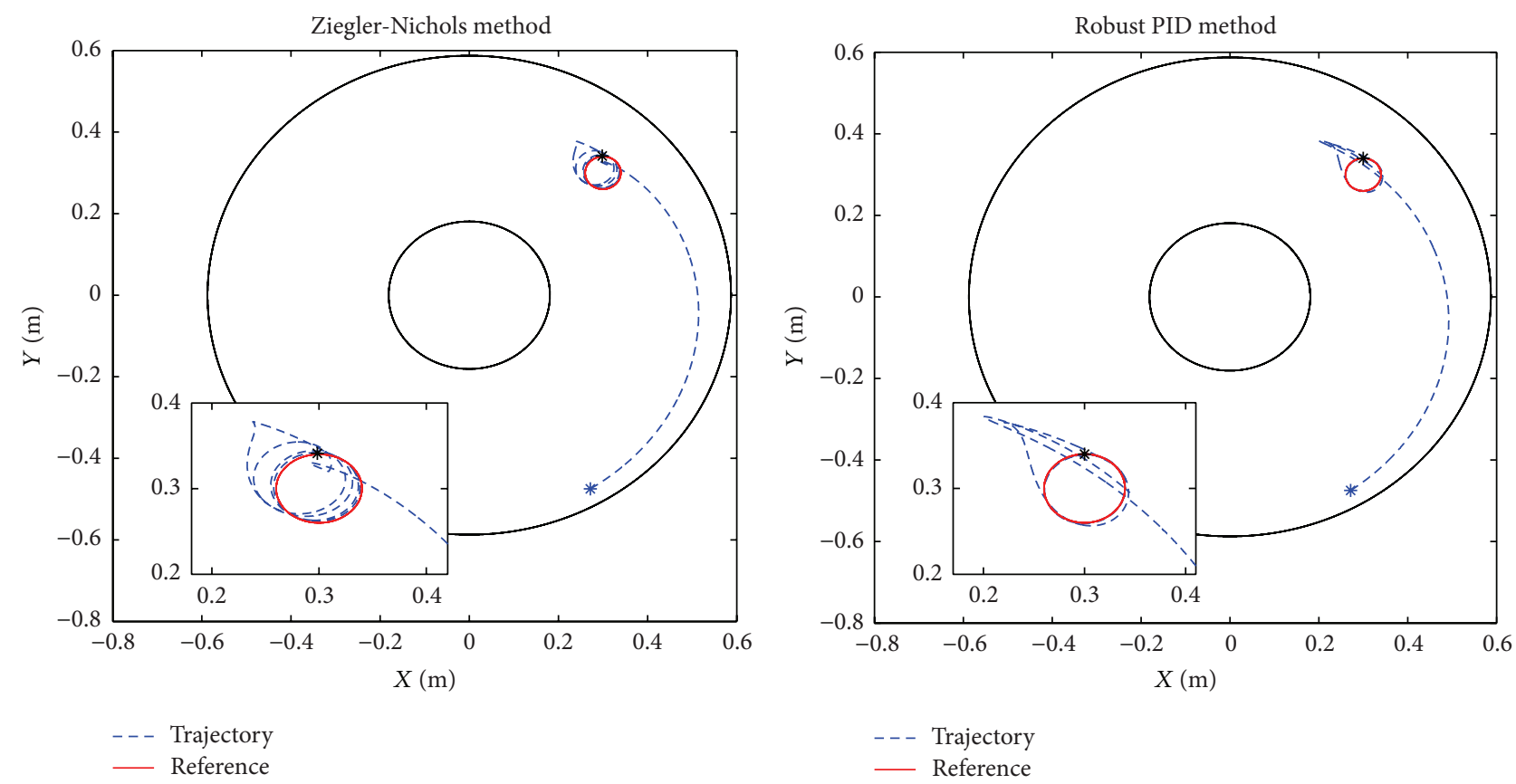

FIGURE 4: Trajectory on work space, comparison between the Ziegler-Nichols method and the Robust PID method.

where the friction vector forces include a low (the term low friction is defined when the friction coefficients belongs in the set $(0,1))$ Coulomb and Viscose friction, $\mathbf{F}_{r}(x) \in \mathbb{R}^{n}$. The initial condition and the control objective are the same as those we present in the previous control shape. Notice that the dissipative vector forces $\mathbf{F}_{r}(x)$ belong in the class of quasi-Lipschitz functions; see Definition 1. For the external disturbances rejection, we introduce a disturbance at time $t=$ 10 seconds. The perturbation structure is as follows:

$$
\begin{gathered}
\varkappa(t)=\left[-0.3 \chi_{t},-0.3 \chi_{t}\right]^{\top}, \\
\chi_{t}:=1\left(t-t_{\mathbf{P}_{1}}\right)-1\left(t-t_{\mathbf{P}_{2}}\right),
\end{gathered}
$$


where the function $u\left(t-t_{k}\right)$ is the characteristic function at time $t_{k}$ [20]. This considered disturbance is because, from the experimental point of view, it introduces a significant deviation than the switched parameter value. For example, if there exist a change of some link mass, it implies a change on the center of mass of each link. Then, the LNDS with dissipative forces (20) can be rewrite as the perturbed form (8). Here $f_{1}(x, t)$ belongs in the quasi-Lipscitz nonlinear functions (6) with

$$
\zeta(x, t)=\varkappa(t)-\mathbf{F}_{r}(x)
$$

and the constants $\delta_{1}=0.023$ and $\delta_{2}=0.26$. The control process is given by regulation of the desired reference, and this one is given by the Heaviside step function given by the following equation:

$$
H(t):= \begin{cases}1 & \text { if } t \geq 0 \\ 0 & \text { if } t<0\end{cases}
$$

moreover, for the control point of view, we implement a regulation point-to-point at the work space. The first point to go is at $\mathbf{P}_{x_{1}}=0.34$ and $\mathbf{P}_{y_{1}}=0.3$ meters at time interval $t \in\left[0, t_{\mathbf{P}_{1}}\right)$ seconds, the second follows a trajectory $\mathbf{P}_{x_{2}}=$ $0.3+.04 \cos (\omega t), \mathbf{P}_{y_{2}}=0.3+.04 \sin (\omega t)$ with $\omega=4 \pi$ for time interval $t \in\left[t_{\mathbf{P}_{1}}, 50\right), \mathbf{P}_{x_{3}}=0.3$, respectively, and in the time $t=25$ seconds, we induce an external perturbation $\xi(t)$ with a duration of $0.5 \mathrm{~s}$. The initial conditions of the system for both control examples are

$$
x_{0}=\left[-\frac{\pi}{2}, \frac{\pi}{4}, 0,0\right]^{\top} \text {. }
$$

The gain matrices given by method of Zingler-Nichols (to obtain the control gain via Zingler-Nichols method, we take into account the linearization of the first-order mathematical model (2) around the center of the area of the desired trajectory tracking) are

$$
\begin{gathered}
\mathbf{K}_{P}=\left(\begin{array}{cc}
24.56 & 0 \\
0 & 32.77
\end{array}\right), \quad \mathbf{K}_{D}=\left(\begin{array}{cc}
28.76 & 0 \\
0 & 29.07
\end{array}\right), \\
\mathbf{K}_{I}=\left(\begin{array}{cc}
10.98 & 0 \\
0 & 14.76
\end{array}\right) .
\end{gathered}
$$

In order to obtain the PID-CT gain matrices, we used the MATLAB Toolbox SeDuMi to solve the corresponding constrained LMI problem (14); see for example [21]. Finally, we obtain the gain matrices as follows:

$$
\begin{gathered}
\mathbf{K}_{P}=1.5 \times\left(\begin{array}{cc}
39.56 & 2.34 \\
2.34 & 28.37
\end{array}\right), \quad \mathbf{K}_{D}=2 \times\left(\begin{array}{cc}
34.63 & 3.3 \\
3.3 & 43.71
\end{array}\right), \\
\mathbf{K}_{I}=2 \times\left(\begin{array}{cc}
1.97 & 0.1 \\
0.1 & 1.96
\end{array}\right) .
\end{gathered}
$$

Figure 2 depicts the evolution of steady-state errors $\tilde{x}$. Figure 4 depicts the control signals $\tau_{1}$ and $\tau_{2}$ for both actuators. Finally, Figure 3 shows how after the induced perturbation the conventional linear PID (red solid line) cannot reject the perturbation, while on the other hand, for the case where the gains were tuned by the proposed methodology (blue solid line), the controller is able to reject the perturbation and to track the desired trajectory.

Clearly, the robust PID control can successfully compensate the uncertainties such as friction, gravity, and other uncertainties of the robot.

\section{Concluding Remarks}

An analysis of global UUB-stability for Lagrangian systems has been presented in this paper. In order to perform the robust stability analysis, we used like-passivity injection based on the dynamical properties of the system and in the characteristics of the quasi-Lipschitz functions. Theoretical robust analysis stability of PID-CT compensator under external but bounded perturbations has been done successfully. Experimental validation was conducted by perturbations between the robotic arm positions $x_{1}$ and $x_{2}$. Here, we have defined a methodology to obtain the $\mathbf{K}_{P}, \mathbf{K}_{D}$, and $\mathbf{K}_{I}$ gains matrices of the PID-CT compensator. Even more, we used an storage function based on the trajectory error in order to guarantee the asymptotic convergence. A comparison between the Ziegler Nichols algorithm and the algorithm proposed showed that it has a better performance in order to reject external disturbances and dynamic uncertainties. Additionally, the maximal overshot of the closed-loop system is given by the ultimate bound of the stability analysis; this is because once the trajectory arrives to the ellipsoid, this remains there. Thus, the main result of this contribution opens the door for the study of output feedback compensators for LNDS. Even more with this result, we will study the case when the dynamical system has not external perturbation and dynamic uncertain, waiting to probe exponential stability (see expression (16)).

\section{Appendix}

\section{Properties of Lagrangian Mechanical Systems}

The dynamic equation for Lagrangian mechanical systems (1) has the following interesting properties. Inertial matrix $\mathbf{D}(q)$ is positive and definite, and $\mathbf{D}(q)$ and $\mathbf{C}(q, \dot{q})$ have the following properties $[7,13,14]$.

(i) There exist some positive constants $\left(\delta_{0}, \delta_{1}<\infty\right)$ such that

$$
\delta_{0} I_{n \times n} \leq \mathbf{D}(q) \leq \delta_{1} I_{n \times n} \quad \forall q \in \mathbb{R}^{n},
$$

where $I_{n \times n}$ denotes the $n$ by $n$ identity matrix. So, matrix $\mathbf{D}^{-1}(q)$ exists and it is positive definite.

(ii) Matrix $\mathbf{C}(q, \dot{q})$ has a relationship with the inertial matrix as follows:

$$
\dot{\mathbf{D}}(q)=\mathbf{C}(q, \dot{q})+\mathbf{C}(q, \dot{q})^{\top} .
$$

In fact, $(1 / 2) \dot{\mathbf{D}}(q)-\mathbf{C}(q, \dot{q})$ is the skew-symmetric property, where $\mathbf{C}(q, \dot{q})$ is a matrix, univocally defined by $\mathbf{D}(q)$. 
Proof of Theorem 3. The time derivative of the energy function $((d / d t) V(y):=\dot{V}(y))$ is given as follows:

$$
\dot{V}(y)=2\langle y, \mathbf{P} \dot{y}\rangle+\left\langle\tilde{x}_{2}, \dot{\mathbf{D}}\left(x_{1}\right) \tilde{x}_{2}\right\rangle
$$

or in extended form

$$
\begin{aligned}
\dot{V}(y)= & \left\langle y,\left[\begin{array}{ccc}
0_{n \times n} & \mathbf{P}_{11}^{\top} & \mathbf{P}_{12} \\
\mathbf{P}_{11} & \mathbf{P}_{12}+\mathbf{P}_{12}^{\top} & \mathbf{P}_{22}^{\top} \\
\mathbf{P}_{12}^{\top} & \mathbf{P}_{22} & 0_{n \times n}
\end{array}\right] y\right\rangle \\
& +2\left\langle\tilde{x}_{2}, \mathbf{D}\left(x_{1}\right) \dot{\tilde{x}}_{2}\right\rangle+\left\langle\tilde{x}_{2}, \dot{\mathbf{D}}\left(x_{1}\right) \tilde{x}_{2}\right\rangle ;
\end{aligned}
$$

notice that, from Lagrangian formulation (3) and from dynamical error, we have

$$
\begin{aligned}
\dot{x}_{2} & =\mathbf{D}^{-1}\left(x_{1}\right)\left\{u+\zeta(x, t)-\mathbf{C}(x) x_{2}-G\left(x_{1}\right)\right\}, \\
\dot{\tilde{x}}_{2} & :=\dot{x}_{2}-\dot{x}_{2}^{d}
\end{aligned}
$$

using the kinetic-Corolis matrices properties (A.2) on the right hand side of (A.3), we have that

$$
\begin{aligned}
& \left\langle\tilde{x}_{2}, \mathbf{D}\left(x_{1}\right) \dot{\tilde{x}}_{2}\right\rangle \\
& =\left\langle\tilde{x}_{2}, \mathbf{D}\left(x_{1}\right)\left(\dot{x}_{2}-\dot{x}_{2}^{d}\right)\right\rangle \\
& =\left\langle\tilde{x}_{2}, u+\zeta(x, t)-\mathbf{C}(x) x_{2}-G\left(x_{1}\right)-\mathbf{D}\left(x_{1}\right) \dot{x}_{2}^{d}\right\rangle \\
& =\left\langle\tilde{x}_{2}, u+\zeta(x, t)-\mathbf{C}(x) \tilde{x}_{2}-\mathbf{C}(x) x_{2}^{d}-G\left(x_{1}\right)\right. \\
& \left.\quad-\mathbf{D}\left(x_{1}\right) \dot{x}_{2}^{d}\right\rangle,
\end{aligned}
$$

and using the control law (9)

$$
\begin{aligned}
u= & \mathbf{D}\left(x_{1}\right) \dot{x}_{2}^{d}+\mathbf{C}(x) x_{2}^{d}+G\left(x_{1}\right)-\mathbf{K}_{I} \xi\left(\tilde{x}_{1}\right) \\
& -\mathbf{K}_{P} \tilde{x}_{1}-\mathbf{K}_{D} \tilde{x}_{2}
\end{aligned}
$$

we obtain the following:

$$
\begin{aligned}
& \left\langle\tilde{x}_{2}, \mathbf{D}\left(x_{1}\right) \dot{\tilde{x}}_{2}\right\rangle \\
& \quad=\left\langle\tilde{x}_{2},-\mathbf{K}_{I} \xi\left(\tilde{x}_{1}\right)-\mathbf{K}_{P} \tilde{x}_{1}-\mathbf{K}_{D} \tilde{x}_{2}-\mathbf{C}\left(x_{1}, x_{2}\right) \tilde{x}_{2}\right\rangle \\
& \quad=\left\langle\tilde{x}_{2},-\mathbf{K}_{I} \xi\left(\tilde{x}_{1}\right)-\mathbf{K}_{P} \tilde{x}_{1}-\mathbf{K}_{D} \tilde{x}_{2}\right\rangle-\left\langle\tilde{x}_{2}, \mathbf{C}(x) \tilde{x}_{2}\right\rangle ;
\end{aligned}
$$

then the time derivative yields as

$$
\begin{aligned}
\dot{V}(y)= & \left\langle y,\left[\begin{array}{ccc}
0_{n \times n} & \mathbf{P}_{11}^{\top} & \mathbf{P}_{12} \\
\mathbf{P}_{11} & \mathbf{P}_{12}+\mathbf{P}_{12}^{\top} & \mathbf{P}_{22}^{\top} \\
\mathbf{P}_{12}^{\top} & \mathbf{P}_{22} & 0_{n \times n}
\end{array}\right] y\right\rangle \\
& +\left\langle\tilde{x}_{2}, \dot{\mathbf{D}}\left(x_{1}\right) \tilde{x}_{2}\right\rangle-2\left\langle\tilde{x}_{2}, \mathbf{C}(x) \tilde{x}_{2}\right\rangle \\
& +2\left\langle\tilde{x}_{2},-\mathbf{K}_{I} \xi\left(\tilde{x}_{1}\right)-\mathbf{K}_{P} \tilde{x}_{1}-\mathbf{K}_{D} \tilde{x}_{2}\right\rangle .
\end{aligned}
$$

This expression contains the skew-symmetric property, and it reduces the expression as follows:

$$
\dot{V}(y)=\left\langle y,\left[\begin{array}{ccc}
0_{n \times n} & \mathbf{P}_{11}^{\top} & \mathbf{P}_{12}-\mathbf{K}_{I}^{\top} \\
\mathbf{P}_{11} & \mathbf{P}_{12}+\mathbf{P}_{12}^{\top} & \mathbf{P}_{22}^{\top}-\mathbf{K}_{P}^{\top} \\
\mathbf{P}_{12}^{\top}-\mathbf{K}_{I} & \mathbf{P}_{22}-\mathbf{K}_{P} & -\mathbf{K}_{D}
\end{array}\right] y\right\rangle
$$

adding and subtracting the energetic function on (A.10), we obtain

$$
\dot{V}(y)=\left\langle y,\left[\begin{array}{ccc}
\alpha \mathbf{P}_{11} & \alpha \mathbf{P}_{12}+\mathbf{P}_{11}^{\top} & \mathbf{P}_{12}-\mathbf{K}_{I}^{\top} \\
\alpha \mathbf{P}_{12}^{\top}+\mathbf{P}_{11} & \alpha \mathbf{P}_{22}+\mathbf{P}_{12}+\mathbf{P}_{12}^{\top} & \mathbf{P}_{22}^{\top}-\mathbf{K}_{P}^{\top} \\
\mathbf{P}_{12}^{\top}-\mathbf{K}_{I} & \mathbf{P}_{22}-\mathbf{K}_{P} & -\mathbf{K}_{D}+\alpha \mathbf{D}\left(x_{1}\right)
\end{array}\right] y\right\rangle-\alpha V(y)
$$

and realizing the same procedure over $\varepsilon\left\|\xi\left(\widetilde{x}_{1}\right)\right\|_{\mathbf{K}_{I}}^{2}$, with $0<\varepsilon$ in the last equation under assumption that

$$
\left\|\xi\left(\tilde{x}_{1}\right)\right\|_{\mathbf{K}_{I}}^{2}:=\xi\left(\tilde{x}_{1}\right)^{\top} \mathbf{K}_{I} \xi\left(\tilde{x}_{1}\right) \leq c_{0}+c_{1}\left\|\xi\left(\tilde{x}_{1}\right)\right\|^{2}
$$

then we have the following expression:

$$
\dot{V}(y) \leq-\langle y, \mathbf{W}(y) y\rangle-\alpha V(y)+\varepsilon c_{0}
$$

where the matrix $\mathbf{W}(y)$ has the following nonlinear-matrix format:

$$
\begin{aligned}
& \mathbf{W}(y) \\
& =\left[\begin{array}{ccc}
-\varepsilon c_{1} \mathbf{I}_{n \times n}+\alpha \mathbf{P}_{11} & \alpha \mathbf{P}_{12}+\mathbf{P}_{11}^{\top} & \mathbf{P}_{12}-\mathbf{K}_{I}^{\top} \\
\alpha \mathbf{P}_{12}^{\top}+\mathbf{P}_{11} & \alpha \mathbf{P}_{22}+\mathbf{P}_{12}+\mathbf{P}_{12}^{\top} & \mathbf{P}_{22}^{\top}-\mathbf{K}_{P}^{\top} \\
\mathbf{P}_{12}^{\top}-\mathbf{K}_{I} & \mathbf{P}_{22}-\mathbf{K}_{P} & -\mathbf{K}_{D}+\alpha \mathbf{D}\left(x_{1}\right)
\end{array}\right] .
\end{aligned}
$$


Now, if we apply the upper bounds properties from the Lagrangian formulation (A.1), this nonlinear matrix becomes a bilinear one $\mathbf{W}$ (see the expression (14)). Then, the energy function time's derivative can be defined as follows:

$$
\dot{V}(y) \leq-\langle y, \mathbf{W} y\rangle-\alpha V(y)+\beta, \quad \beta:=\varepsilon c_{0} ;
$$

note that if the matrix $\mathbf{W}$ is positive and definite, then the energetic function time's derivative (A.3) holds the following inequality:

$$
\dot{V}(y) \leq-\alpha V(y)+\beta
$$

and its solution on the time interval $t \in\left[t_{0}, \mathbf{t}\right)$ defines the next inequality (the variable $\mathbf{t}$ defines a funny change of variable on time, and $V(0)$ defines the initial condition of the energetic function):

$$
\begin{aligned}
\dot{V}(y) \leq & -\alpha V(y)+\beta \\
\Longrightarrow & V(y) \leq V(0) \exp \left[-\alpha\left(t-t_{0}\right)\right] \\
& +\frac{\beta}{\alpha}\left(1-\exp \left[-\alpha\left(t-t_{0}\right)\right]\right)
\end{aligned}
$$

and its final value comes to the expression (15), which means that all trajectories of the extended vector $\{y\}$ converge to an attractive ellipsoid. The proof is complete.

Proof of the Theorem 6. Notice that the function $[\bar{y}]_{+}$is not differentiable in the point $\bar{y}=0$, but the function $\left([\bar{y}]_{+}\right)^{2}$ is differentiable everywhere. Based on this fact and following [22], we may conclude that the function

$$
G(V(y)):=\left(\left[\sqrt{V(y)}-\sqrt{\frac{\beta}{\alpha}}\right]_{+}\right)^{2}
$$

is also differentiable everywhere. That is because

$$
\begin{aligned}
\frac{d}{d t} G(V(y)) & =\frac{d}{d V} G(V(y)) \dot{V}(y) \\
& =\left[\sqrt{V(y)}-\sqrt{\frac{\beta}{\alpha}}\right]_{+} \frac{\dot{V}(y)}{\sqrt{V(y)}} .
\end{aligned}
$$

Using the inequality (A.16), we obtain

$$
\begin{aligned}
\frac{d}{d t} G(V(y)) \leq & {\left[\sqrt{V(y)}-\sqrt{\frac{\beta}{\alpha}}\right]_{+} \frac{-\alpha V(y)+\beta}{\sqrt{V(y)}} } \\
& =-\alpha\left[\sqrt{V(y)}-\sqrt{\frac{\beta}{\alpha}}\right]_{+} \frac{V(y)-\beta / \alpha}{\sqrt{V(y)}} \\
& =-\alpha\left(\left[\sqrt{V(y)}-\sqrt{\frac{\beta}{\alpha}}\right]_{+}\right)^{2} \\
& \cdot \frac{\sqrt{V(y)}+\sqrt{\beta / \alpha}}{\sqrt{V(y)}} \leq 0
\end{aligned}
$$

which proves (18). Since the nonnegative function $G(V(y))$ is monotonically nonincreasing, by the Weierstrass theorem, it has a limit; that is, there exists

$$
0 \leq G^{*}=\lim _{t \rightarrow \infty} G(V(y)) .
$$

Integrating (A.20) implies that

$$
\begin{gathered}
G(V(y))-G(V(y)) \leq-\alpha \int_{=0}^{T}\left(\left[\sqrt{V(y(\mathbf{t}))}-\sqrt{\left.\left.\frac{\beta}{\alpha}\right]_{+}\right)^{2}}\right.\right. \\
\cdot \frac{\sqrt{V(y(\mathbf{t}))}+\sqrt{\beta / \alpha}}{\sqrt{V(y(\mathbf{t}))}} d \mathbf{t}
\end{gathered}
$$

or equivalently that

$$
\begin{aligned}
& \alpha \int_{\mathbf{t}=0}^{\top}\left(\left[\sqrt{V(y(\mathbf{t}))}-\sqrt{\frac{\beta}{\alpha}}\right]_{+}\right)^{2}\left(1+\frac{\sqrt{\beta / \alpha}}{\sqrt{V(y(\mathbf{t}))}}\right) d \mathbf{t} \\
& \quad \leq G(V(y(0)))-G(V(y(t))) \leq G(V(y(0))) \\
& \quad=\text { const }
\end{aligned}
$$

which, for $t \rightarrow \infty$ and in view of (A.18), leads to the following conclusion:

$$
\int_{t=0}^{\infty} G(V(y(\mathbf{t})))\left(1+\frac{\sqrt{\beta / \alpha}}{\sqrt{V(t(\mathbf{t}))}}\right) d \mathbf{t}<\infty .
$$

The convergence of the last integral permits to state that there exists a subsequence $\left\{t_{k}\right\}_{k=1,2, \ldots}$ such that $G\left(V\left(y\left(t_{k}\right)\right)\right) \underset{k \rightarrow \infty}{\rightarrow}$ 0 . But the sequence $G(V(y(t)))$ converges (see (A.21)), and hence all its subsequences have the same limit point, which proves that $G^{*}=0$, and, as a result, we have that $\left\{x \in \mathbb{R}^{n}\right.$ : $G(V(y))=0\}$ is a positive invariant ellipsoid, and then the proof is complete.

\section{Conflict of Interests}

The authors declare that there is no conflict of interests regarding the publication of this paper.

\section{References}

[1] V. I. Arnold, Mathematical Methods of Classical Mechanics, vol. 60 of Graduate Texts in Mathematics, Springer, New York, NY, USA, 2nd edition, 1989.

[2] F. Lewis, D. Dawson, and C. Abdallah, Robot Manipulator Control: Theory and Practice, Marcel Dekker, New York, NY, USA, 2nd edition, 2004.

[3] J. LaSalle and S. Lefschetz, Stability by Liapunov's Direct Method: With Applications, Academic Press, New York, NY, USA, 1961.

[4] W. Haddad and V. Chellaboina, Nonlinear Dynamical Systems and Control: A Lyapunov-Based Approach, Princeton University Press, Princeton, NJ, USA, 2008. 
[5] T. Taniguchi, "Stability theorems of perturbed linear ordinary differential equations," Journal of Mathematical Analysis and Applications, vol. 149, no. 2, pp. 583-598, 1990.

[6] V. Parra-Vega, S. Arimoto, Y.-H. Liu, G. Hirzinger, and P. Akella, "Dynamic sliding PID control for tracking of robot manipulators: theory and experiments," IEEE Transactions on Robotics and Automation, vol. 19, no. 6, pp. 967-976, 2003.

[7] M. Spong, S. Hutchinson, and M. Vidyasagar, Robot Modeling and Control, John Wiley \& Sons, 2006.

[8] R. Ortega, A. Astolfi, and N. E. Barabanov, "Nonlinear PI control of uncertain systems: an alternative to parameter adaptation," Systems \& Control Letters, vol. 47, no. 3, pp. 259-278, 2002.

[9] A. A. Pervozvanski and L. B. Freidovich, "Robust stabilization of robotic manipulators by PID controllers," Dynamics and Control, vol. 9, no. 3, pp. 203-222, 1999.

[10] T.-Y. Kuc and W.-G. Han, "An adaptive PID learning control of robot manipulators," Automatica, vol. 36, no. 5, pp. 717-725, 2000.

[11] K. J. Åström and T. Hägglund, "Revisiting the Ziegler-Nichols step response method for PID control," Journal of Process Control, vol. 14, no. 6, pp. 635-650, 2004.

[12] K. J. Åström and T. Hägglund, Advanced PID Control, Instrumentation, Systems, and Automation Society (ISA), Long Beach, Calif, USA, 2006.

[13] R. Kelly, V. S. Davila, and A. Loría, Control of Robot Manipulators in Joint Space, Springer, London, UK, 2005.

[14] Bullo and Lewis, Geometric Control of Mechanical Systems, Springer, Berlin, Germany, 2004.

[15] F. Bullo and R. M. Murray, "Tracking for fully actuated mechanical systems: a geometric framework," Automatica, vol. 35, no. 1, pp. 17-34, 1999.

[16] J. D. Glover and F. C. Schweppe, "Control of linear dynamic systems with set constrained disturbances," IEEE Transactions on Automatic Control, vol. 16, no. 5, pp. 411-423, 1971.

[17] M. Van, H.-J. Kang, Y.-S. Suh, and K.-S. Shin, "A robust fault diagnosis and accommodation scheme for robot manipulators," International Journal of Control, Automation and Systems, vol. 11, no. 2, pp. 377-388, 2013.

[18] A. B. Kurzhanski and V. M. Veliov, Modeling Techniques and Uncertain Systems, Birkhäuser, New York, NY, USA, 1994.

[19] P. Ordaz and A. Poznyak, "KL'-gain adaptation for attractive ellipsoid method," IMA Journal of Mathematical Control and Information, 2014.

[20] J. E. Marsden, Elementary Classical Analysis, W. H. Freeman, 1974.

[21] D. Peaucelle, D. Henrion, Y. Labit, and K. Taitz, User's Guide for SeDuMi Interface 1.04, 2002.

[22] A. Poznyak, Variable Structure Systems: from Principles to Implementation, vol. 66 of IEE Control Engineering, chapter 3, 2004. 


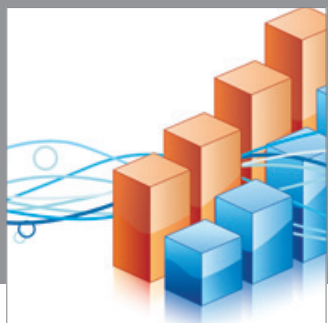

Advances in

Operations Research

mansans

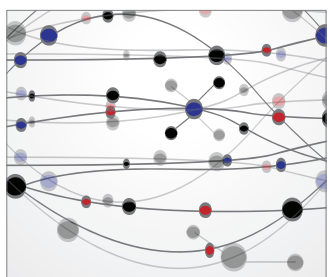

The Scientific World Journal
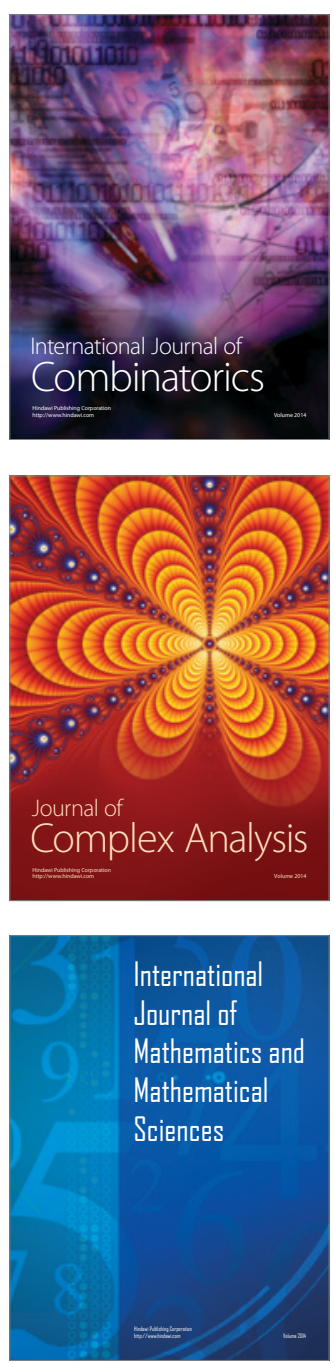
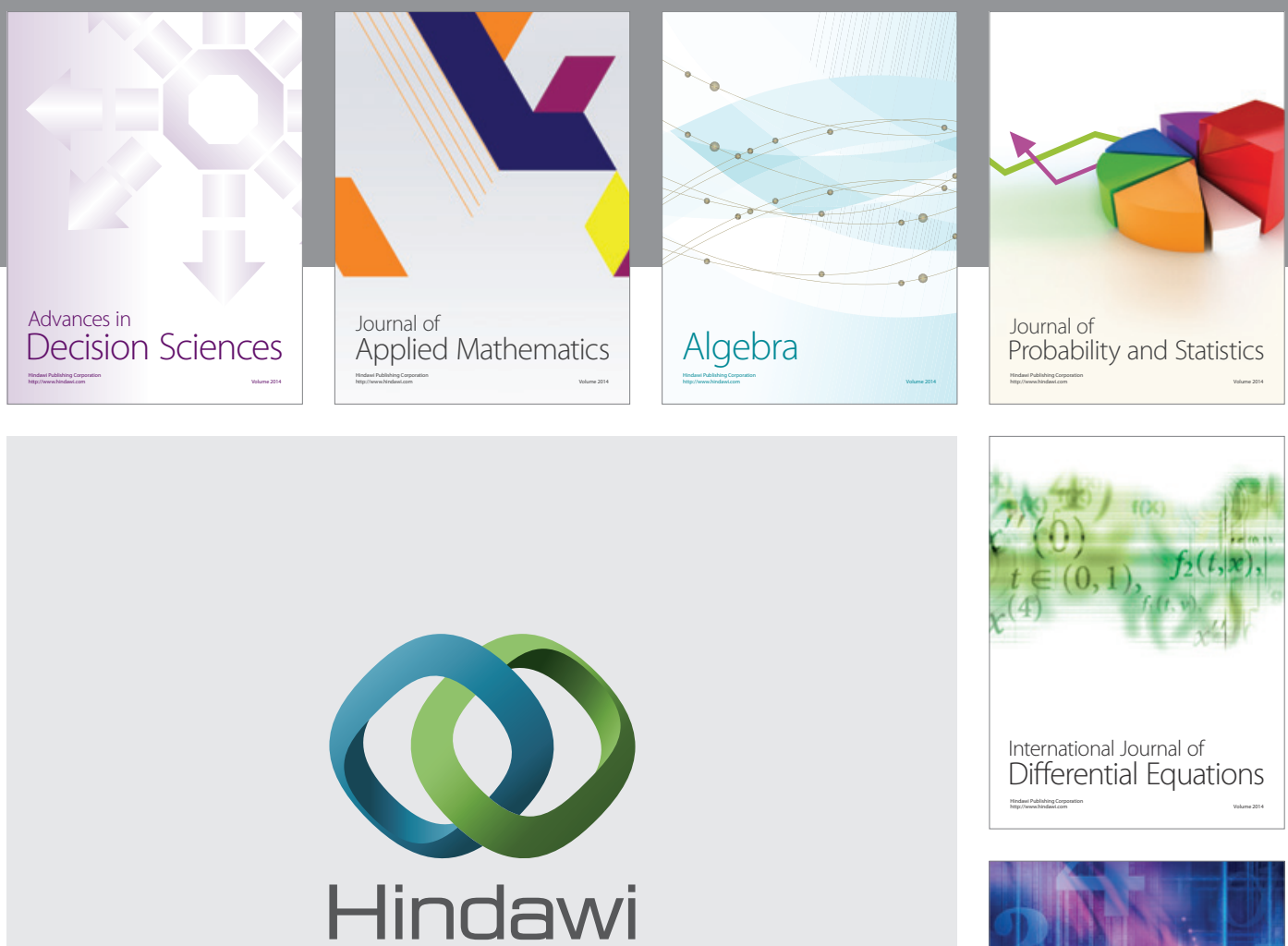

Submit your manuscripts at http://www.hindawi.com
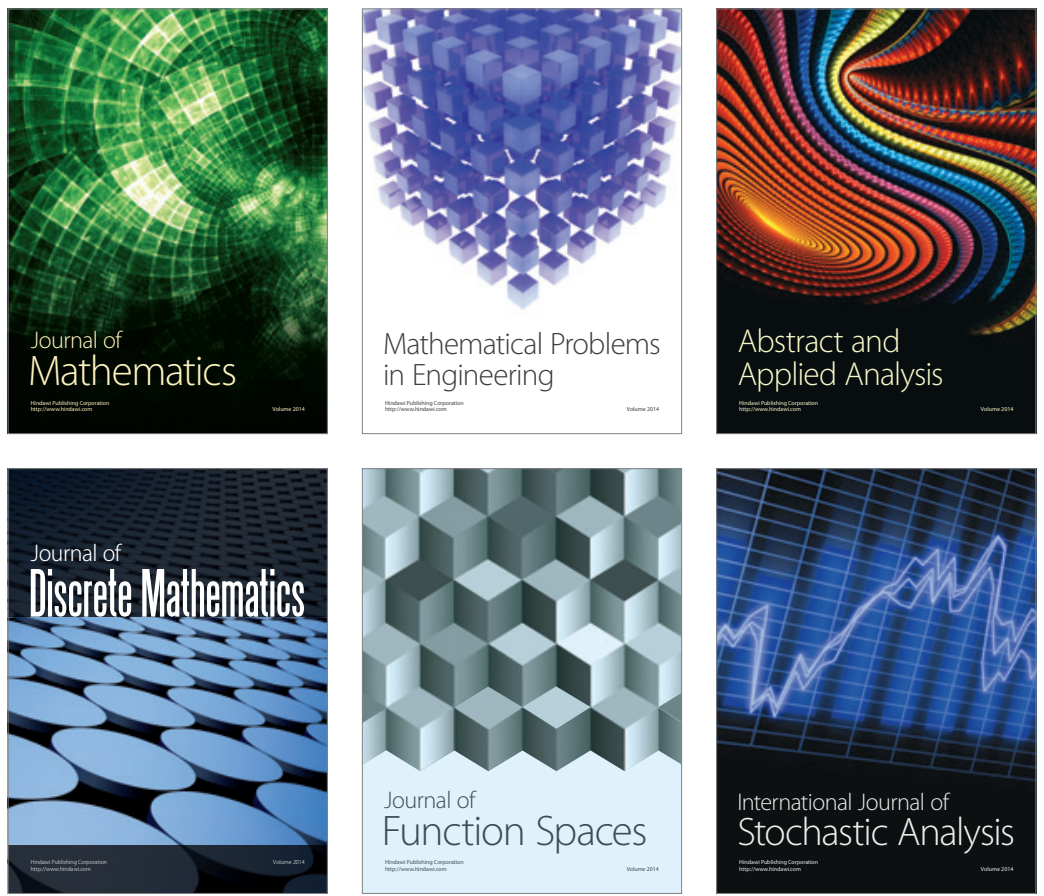

Journal of

Function Spaces

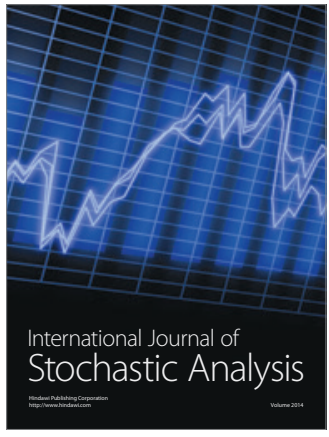

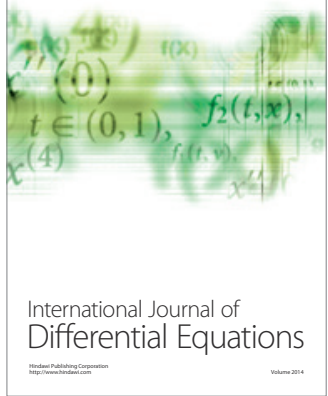
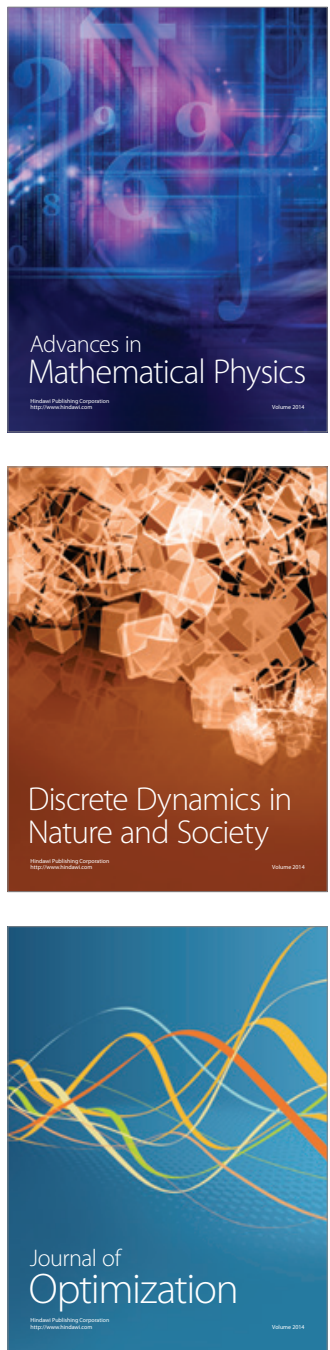